\title{
Philosophiques
}

\section{Pour un bilan culturel du Nouveau Monde}

\section{Guy Godin}

Volume 11, numéro 1, avril 1984

URI : https://id.erudit.org/iderudit/203249ar

DOI : https://doi.org/10.7202/203249ar

Aller au sommaire du numéro

Éditeur(s)

Société de philosophie du Québec

ISSN

0316-2923 (imprimé)

1492-1391 (numérique)

Découvrir la revue

Citer ce document

Godin, G. (1984). Pour un bilan culturel du Nouveau Monde. Philosophiques, 11(1), 185-190. https://doi.org/10.7202/203249ar d'utilisation que vous pouvez consulter en ligne.

https://apropos.erudit.org/fr/usagers/politique-dutilisation/ 


\title{
POUR UN BILAN CULTUREL DU NOUVEAU MONDE
}

\author{
par Guy Godin
}

À la question posée : Le Nouveau Monde : héritage ou création ? ${ }^{1}$ Je réponds par une affirmation qui change les termes de l'alternative : Le Nouveau Monde : dépassement et décadence. Je propose ainsi une lecture personnelle de la situation sous la forme d'un bilan dont je suis conscient qu'il n'est pas complet mais que je formule de façon péremptoirement universelle pour forcer l'attention sur des points qui me paraissent centraux.

Caractériser Le Nouveau Monde comme dépassement et décadence par rapport à l'Ancien, c'est mettre l'accent sur le fait que le Nouveau Monde est en état de crise, à un point de rupture qui, pour prendre une image mathématique, pourrait se décrire comme la présence simultanée sur un même axe vertical du sommet et du creux de la vague. Cette définition de la crise en vaut une autre; elle a au moins le mérite de faire une large place à la contradiction dont les matérialismes dialectique et historique nous ont appris qu'elle était le moteur de l'histoire.

Le Monde Antique a péri d'une contradiction semblable entre le « miracle grec » et « l'empire romain ». Le génie du premier tenait au raffinement de la pensée et de la parole dont l'élan naturel poussait à ce que nous appelons aujourd'hui l'élitisme. Assumées par le génie actif et organisateur du second, les réalisations du premier ont été entraînées sur la pente de la vulgarité et, paradoxalement, vers l'égalitarisme de ce que nous

1. Ce texte reproduit une communication que les organisateurs du XVII ${ }^{e}$ Congrès mondial de philosophie n'ont pas soumis à la lecture parce qu'une autre communication du même auteur avait déjà été acceptée. 
appelons aujourd'hui la démocratie. L'explosion se répercuta pendant des siècles. Faut-il en chercher la cause dans quelque "loi de l'histoire " ou bien dans une conjoncture particulière au Monde Antique selon laquelle Sapiens aurait eu tort de précéder Faber? Au temps de ce qu'on a appelé la Renaissance, le Monde Occidental a recollé certains fragments de l'éclatement ancien en leur mêlant deux nouveaux ingrédients dont le potentiel explosif n'était pas moindre : les valeurs chrétiennes qui avaient créé un autre monde dans l'entre-temps, et celles d'une science naissante qui déjà montrait un fort appétit ${ }^{2}$. On découvrait aussi le Nouveau Monde, qui allait devenir partie intégrante de l'Occident Moderne et qui, dans une symbiose parfois mouvementée avec l'Ancien, devait porter tout ce brassage au point critique que nous vivons. Il se prépare une mutation profonde, sinon un nouvel éclatement, dont la figure se laisse à peine deviner, tant il est vrai qu'il est plus facile de se construire un passé à son image que de préparer un avenir conforme à ses désirs. Les signes des temps laissent croire que c'est Faber qui prépare sa revanche; le technologisme issu de la science veut anéantir jusqu'aux traces des efforts que le monde chrétien a déployés dans sa recherche d'une sagesse extra-mondaine.

Mais la façon dont a été formulée la question à laquelle nous voulons apporter des éléments de réponse a peut-être engagé notre réflexion sur une mauvaise piste. S'il est difficile d'analyser la culture du Nouveau Monde vu comme entité séparable de l'Occident, il est devenu impossible de considérer l'Occident lui-même comme séparé du reste du monde. La prise de conscience de ce fait constitue l'un des facteurs de la crise présente, principalement par ce phénomène de tendance à la " mondialisation » de la culture que le développement accéléré des moyens de communication a fait naître. Pour rendre justice à la question posée, il faudrait sans doute en élargir la perspective d'une façon qui dépasserait le cadre de ce travail. Réduisons-la plutôt à deux questions aussi péremptoirement particulières que ma première réponse fut universelle : 1) Einstein était-il américain ? 2) Jusqu'à quel point l'américan way of life a-t-il influencé, par le tru-

2. Je ne mentionne pas le capitalisme, car il fait partie des valeurs chrétiennes. On pourrait d'ailleurs en dire autant du marxisme. 
chement entre autres du coca-cola, la culture des Caingang de la Sierra da Fartura (petit groupe humain qui est sans doute le plus éloigné de tout ce que connaît le monde occidental) ${ }^{3}$ ?

Poser la première question c'est du même coup démasquer le racisme simpliste dont témoignerait celui qui voudrait faire le bilan culturel de la science par une analyse statistique de la provenance des grands savants et des prix Nobel. La science n'a pas de frontières même si elle est très dépendante de " conditions locales ». Mais on peut constater que la contribution du Nouveau Monde à ce mouvement qui a conduit la science à ses sommets a consisté principalement à rendre présents dans les temps modernes de l'Occident le climat nouveau d'une liberté jeune et non encore assujettie au poids d'une longue tradition, ainsi que l'abondance de ressources dont l'ampleur est à la mesure de l'immensité des espaces.

Le choix d'Einstein pour ma question n'est pas arbitraire mais symbolique. Les sommets atteints par la science constituent un dépassement des ambitions originelles mais dans un sens qui est tout autre que celui des prétentions d'un scientisme simpliste : la sciencen'a pas trouvé réponse à tout mais elle a acquis une maîtrise exceptionnelle dans la façon de poser les questions. Elle a perfectionné sa vision du monde et de l'homme en développant sans scrupule cette méthode d'analyse qui consiste à décomposer les objets pour mieux les pénétrer et en exploitant à fond les virtualités de l'expérience comme moyen de vérification d'une part, et celles de la mathématique comme moyen de théorisation et de prédiction d'autre part. Le dernier perfectionnement de cette méthode générale a consisté à y introduire de façon opératoire la dimension temporelle comme Einstein l'a fait dans le domaine particulier de la physique. L'objet n'est plus seulement vu comme une chose à décomposer qui est là tout entière devant nous dans l'espace, mais comme une chose à saisir dans le déroulement même de sa succession temporelle. On veut le cerner de toutes parts, le poursuivre jusque dans ses moindres replis et dans toutes les dimensions qui conditionnent sa présence dans le monde, et même dans celles qui ne sont pas

3. Cf. J.-W. Lapierre, Essai sur le fondement du pouvoir politique, Aixen-Provence, Éditions Ophrys, 1968, p. 496-506. 
encore. À l'origine, il y a l'idée d'évolution à laquelle la découverte du Nouveau Monde est loin d'être étrangère. Einstein en a généralisé la portée jusqu'aux aspects méthodologiques de la science. Les sciences humaines ont commencé à adapter ce modèle à leur objet dans une approche où la linguistique et ses dépendants jouent un rôle semblable à celui de la mathématique tandis que l'histoire et les diverses analyses des discours font le pendant de l'expérience. Au-delà des modes passagères et successives, qui périodiquement, tendent à exagérer l'importance de telle ou telle méthode, il reste que les discours de l'homme donnent un accès au moins partiel aux lois de son comportement et de șa pensée, comme l'expérience aux secrets de la nature.

Toutefois, les limites rencontrées dans l'application de cette méthode aux deux frontières opposées de l'infiniment grand et de l'infiniment petit ont fait surgir des mises en question que diverses tentatives de réajustement n'ont pas vraiment réussi à calmer de telle sorte qu'on peut prévoir un remaniement en profondeur de la notion de science, la présente ayant épuisé ses possibilités de développement. Cependant elle n'a pas encore totalement épuisé son pouvoir de réducteur, non seulement en ce qui regarde son intolérance plus ou moins avouée vis-à-vis toute autre forme de savoir, mais aussi en ce qui concerne les valeurs, dont la présence en l'homme rejaillit inévitablement sur le monde. Non seulement la science a-t-elle du mal à se protéger des idéologies externes, elle est de plus en plus aux prises avec ses idéologies internes.

L'allusion aux valeurs nous conduit à notre deuxième question, l'ethos se reliant au logos par le biais de la technè dont d'autres ont déjà dit tous les bienfaits et tous les méfaits, le Nouveau Monde ayant sans doute le monopole des uns et des autres grâce à l'immensité des ressources quı en ont permis la prolifération. L'aspect extérieur le plus frappant du Nouveau Monde est sans doute l'abondance des merveilles techniques utilisées couramment par les Américains dans le quotidien. Cette omniprésence de la technique caractérise une civilisation et sécrète ses propres valeurs ; mais ce n'est pas là l'essentiel car ces valeurs sont ellesmêmes "finalisées" par celles plus profondes et englobantes qu'impose la domination de la sphère économique dans un monde axé sur la croissance indéfinie par le développement exacerbé de 
la productivité. Le Nouveau Monde a fait ici une expérience inédite - et qui n'était peut-être pas possible ailleurs, celle de pousser à bout dans toute la rigueur de sa logique originelle l'idée de la « richesse des nations" - avec des moyens que n'avait pas prévus l'inventeur - en créant un état social d'abondance qui a fait accéder la masse à un niveau de vie sans précédent. Le minimum vital nécessaire au bonheur imaginé autrefois par une scolastique abstraite dans un climat de pénurie est devenu une réalité concrète. Et pourtant, au bout du compte, il n'est pas nécessaire de faire appel aux prophètes de malheur pour constater l'insatisfaction et la crise imminente.

Là aussi des sommets ont été atteints. Des valeurs humaines, sociales, intellectuelles, littéraires, artistiques, etc. autrefois réservées au petit nombre ont été mises à la disposition de tous dans un climat de spontanéité, de facilité, de liberté, d'enthousiasme et de respect d'autrui, faisant l'envie des peuples aux prises avec les dictatures politiques ou la misère économique. $\mathrm{Au}$ moment où ce modèle tend à être copié un peu partout, il semble en voie de se dissoudre de l'intérieur. La cause en seraitelle dans une crise de croissance d'un peuple encore jeune en passe de devenir adulte ? Ou serait-ce la fatalité historique qui a démontré l'inéluctable récurrence des crises économiques ? Ou encore la perte de vitesse face à la montée d'autres puissances économiques et culturelles ? Tous ces facteurs ont sans doute joué, et beaucoup d'autres. Mais le ressort profond est à rechercher dans ces cupidités individuelles que la "main invisible " a été impuissante à harmoniser ; dès le départ étaient présents en germe, et le dépassement, et la chute.

Comme l'a bien vu Hannah Arendt ${ }^{4}$, ce n'est pas la revanche de Faber qui se prépare mais c'est déjà le commencement du triomphe de l'Homo laborans. Celui-ci n'a qu'un motif : la vie comme souverain bien, et qu'un moyen : le cycle infernal de la consommation. L'agent réel de ce triomphe n'est ni la masse laborieuse, ni la plus-value arrachée au «travail social moyen abstrait " mais ce que j'appellerais le détournement produit par le spéculatif moyen abstrait ${ }^{5}$. Aristote avait déjà compris que,

4. Cf. La condition de l'homme moderne, Paris, Calmann-Lévy, 1961, p. 360.

5. En m'excusant auprès de mes collègues aristotélico-thomistes de cet usage du mot spéculatif. 
contrairement au bien-vivre (aujourd'hui : qualité de la vie), la vie tout court fonde un désir infini, emporté et aveugle à tout le reste. La montée de la violence gratuite dans le monde témoigne du fait que ce désir est plus particulièrement aveugle à la vie de l'autre. Un passage de la Bible avait déjà exprimé cette idée dans cette formule lapidaire : mieux vaut un chien vivant qu'un lion mort. La variante contemporaine pourrait se formuler ainsi : le cadavre a toujours tort.

Faculté de philosophie

Université Laval 